\title{
Diffuse Large B-Cell Lymphoma Arising within Ileal Neobladder: An Expanding Spectrum of Diffuse Large B-Cell Lymphoma Associated with Chronic Inflammation
}

\author{
Hyekyung Lee, MD, $\mathrm{PhD}$ \\ Hyunbin Shin, MD2 \\ Nae Yu Kim, MD ${ }^{3}$ \\ Hyun Sik Park, MD2 \\ Jinsung Park, MD, PhD²
}

Departments of ${ }^{1}$ Pathology, ${ }^{2}$ Urology, and ${ }^{3}$ Internal Medicine, Eulji University Hospital, Daejeon, Korea
Correspondence: Jinsung Park, MD, PhD Department of Urology, Eulji University Hospital, Eulji University School of Medicine, 95 Dunsanseoro, Seo-gu, Daejeon 35233, Korea Tel: 82-42-611-3533

Fax: 82-42-259-1111

E-mail: jspark.uro@gmail.com

Received January 10, 2019

Accepted March 21, 2019

Published Online March 22, 2019
Diffuse large B-cell lymphoma associated with chronic inflammation (DLBCL-Cl), specifically arising in ileal neobladder, is a rare neoplasm. We present an unusual case of EpsteinBarr virus (EBV)-positive DLBCL-Cl arising within neobladder with detailed clinical, histological, and immunophenotypical features in an immunocompetent patient. An 88-year-old male was admitted for gross hematuria. He had undergone radical cystectomy and ileal neobladder 17 years ago for invasive bladder cancer. Computed tomography showed enhancing lesions on dome and posterior wall of neobladder with mucosal thickening and multiple enlarged retroperitoneal lymphadenopathies. Transurethral resection of neobladder lesion revealed the diffuse infiltration of large lymphoid cells which were positive for CD20, CD30, and multiple myeloma oncogen-1 with EBV-encoded small RNAs co-localizing, and diagnosis of EBV-positive DLBCL-Cl was made. After multi-agent chemotherapy, the lesion disappeared. We suggest that clinicians should consider the possibility of DLBCL-Cl in patients presented with hematuria during follow-up after bladder reconstruction.

\section{Key words}

Diffuse large B-cell lymphoma, Epstein-Barr virus, lleal neobladder, Bladder reconstruction, Radical cystectomy

\section{Introduction}

Diffuse large B-cell lymphoma with chronic inflammation (DLBCL-CI) is a lymphoid neoplasm occurring in the setting of longstanding chronic inflammation and showing association with Epstein-Barr virus (EBV) [1]. The development of malignant lymphoma in a reconstructed organ, such as the stoma, ileal conduit, or ileal neobladder, is extremely rare $[2,3]$. Most cases involve body cavities or narrow spaces, while pyothorax-associated lymphoma is the prototypical form [4]. In this article, we report a case of a patient with EBV-positive DLBCL-CI arising within ileal neobladder. 

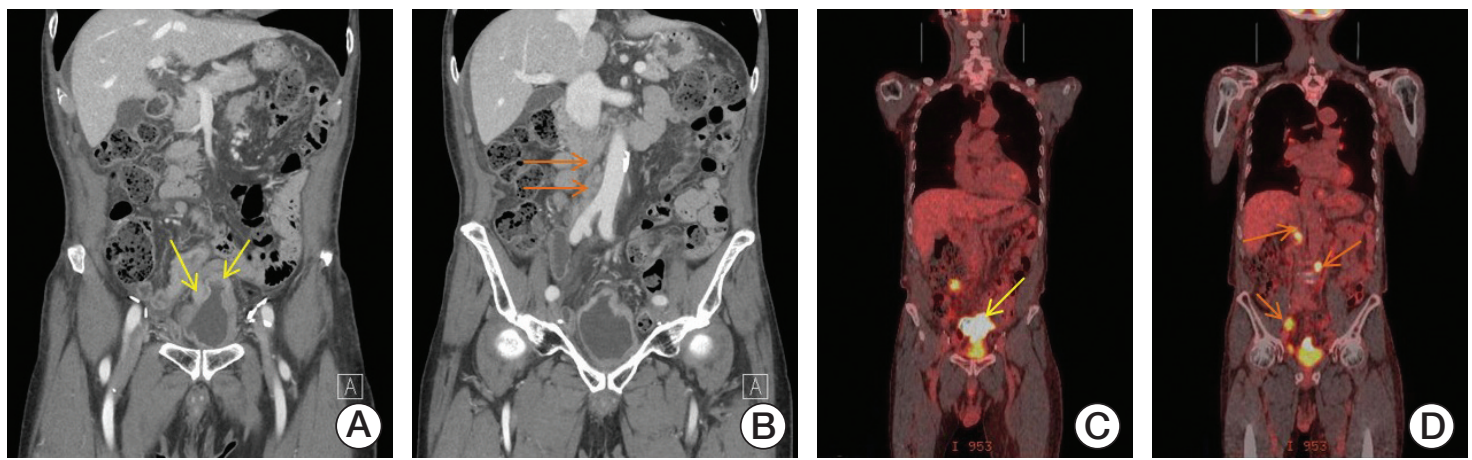

Fig. 1. Computed tomography (CT) scan showed enhancing lesion in the dome and posterior wall of ileal neobladder (yellow arrows) (A) and multiple retroperitoneal lymphadenopathies (orange arrows) (B). Positron emission tomography computed tomography showed localized ${ }^{18} \mathrm{~F}$-fluorodeoxyglucose accumulation in ileal neobladder (yellow arrow) (C) and multiple retroperitoneal lymph nodes (orange arrows) (D).

\section{Case Report}

An 88-year-old immunocompetent man was admitted with gross hematuria for several days. He had a history of invasive bladder cancer and had received curative radical cystectomy and ileal neobladder reconstruction 17 years ago. The patient did not have any other symptoms, including fever, night sweats, weight loss or fatigue. He had neither a history of inflammatory bowel disease nor medication of immunosuppressant. As initial diagnostic work-ups for hematuria, computed tomography (CT) scan showed enhancing lesion on dome and posterior wall of neobladder with mucosal thickening, and multiple enlarged retroperitoneal lymphadenopathies including paraaortic, aortocaval, retrocaval, right common iliac, and ileocolic mesenteric lymph nodes, measuring up to $4 \mathrm{~cm}$ (Fig. 1A and B). Multiple metallic clips, which were used during previous neobladder formation, were noted in pelvic cavity but were distant from the mass lesion. On cystoscopy, diffuse, sessile mucosal lesion was observed on dome and posterior wall, consistent with CT finding. Colonoscopy showed normal colonic mucosa without definite mass formation or abnormal vasculatures. Positron emission tomography (PET)-CT scan revealed hypermetabolic irregular mass measuring about $6.7 \mathrm{~cm}$ in length at dome and posterior wall of neobladder with enlarged hypermetabolic retrocrural, retrocaval, paraaortic, aortocaval, right common iliac, ileocolic lymph nodes measuring up to $4 \mathrm{~cm}$ in diameter (Fig. 1C and D). There were no specific radiologic abnormalities in other organs.

For pathologic diagnosis of mucosal lesion on ileal neobladder, the patient underwent transurethral resection under general anesthesia. The mass lesion was too hard to be obtained by biopsy forceps; thus, the lesion was resected using bipolar resectoscope at multiple sites. The surgery was uneventful without neobladder perforation.

The biopsied samples of ileal neobladder were composed of several fragments of soft tissue measuring $2 \times 1 \times 1 \mathrm{~cm}$ in aggregates. Microscopic examination revealed different histology in each fragment. The severity of infiltration with CD20-positive atypical B lymphocytes was variable from diffuse infiltrates to focal aggregates. The neoplastic cells had large nuclei, prominent nucleoli, irregular nuclear membranes, and scant cytoplasm (Fig. 2A). Angioinvasion by atypical lymphocytes with intravascular thrombosis was noted (Fig. 2B) with high EBV-encoded small RNA 1 and 2 (EBER) positivity (Fig. 2C). The EBER positivity was prominent near ulcerated mucosa and perivascular area. The CD20-positive atypical lymphocytes (Fig. 2D) showed the non-germinal center immunophenotype with negative reaction to CD10, BCL6, but positive reaction to multiple myeloma oncogen-1 (Fig. 2E) with Ki-67 index over 40\% . Patchy CD30 positivity of $10 \%$ (Fig. 2 F) and scattered p53 positivity of $40 \%$ were also noted (Fig. 2G). The chronic inflammation area was noted in a patchy manner within ileal tissue with focal epithelioid histiocytic reaction resembling granuloma and CD3-positive $\mathrm{T}$ cells. Blood tests for EBV antibodies showed that IgG for EBV viral capsid antigen (EBV-VCA) and IgG for Epstein-Barr nuclear antigen were positive, but IgM for EBV-VCA and IgM/IgG for EBV early antigen diffuse and restricted were not detected. EBV DNA was not found in the blood by polymerase chain reaction (PCR) assay. Bone marrow study showed no lymphomatous lesion. Integrating these findings, a diagnosis of DLBCL-CI, staged IIE according to the Ann Arbor Staging system, was made [5,6].

After diagnosis, patient received multi-agent chemotherapy with rituximab $375 \mathrm{mg} / \mathrm{m}^{2} \mathrm{D} 1$ plus cyclophosphamide $750 \mathrm{mg} / \mathrm{m}^{2} \mathrm{D} 1$, doxorubicin $40 \mathrm{mg} / \mathrm{m}^{2} \mathrm{D} 1$, vincristine 1.4 

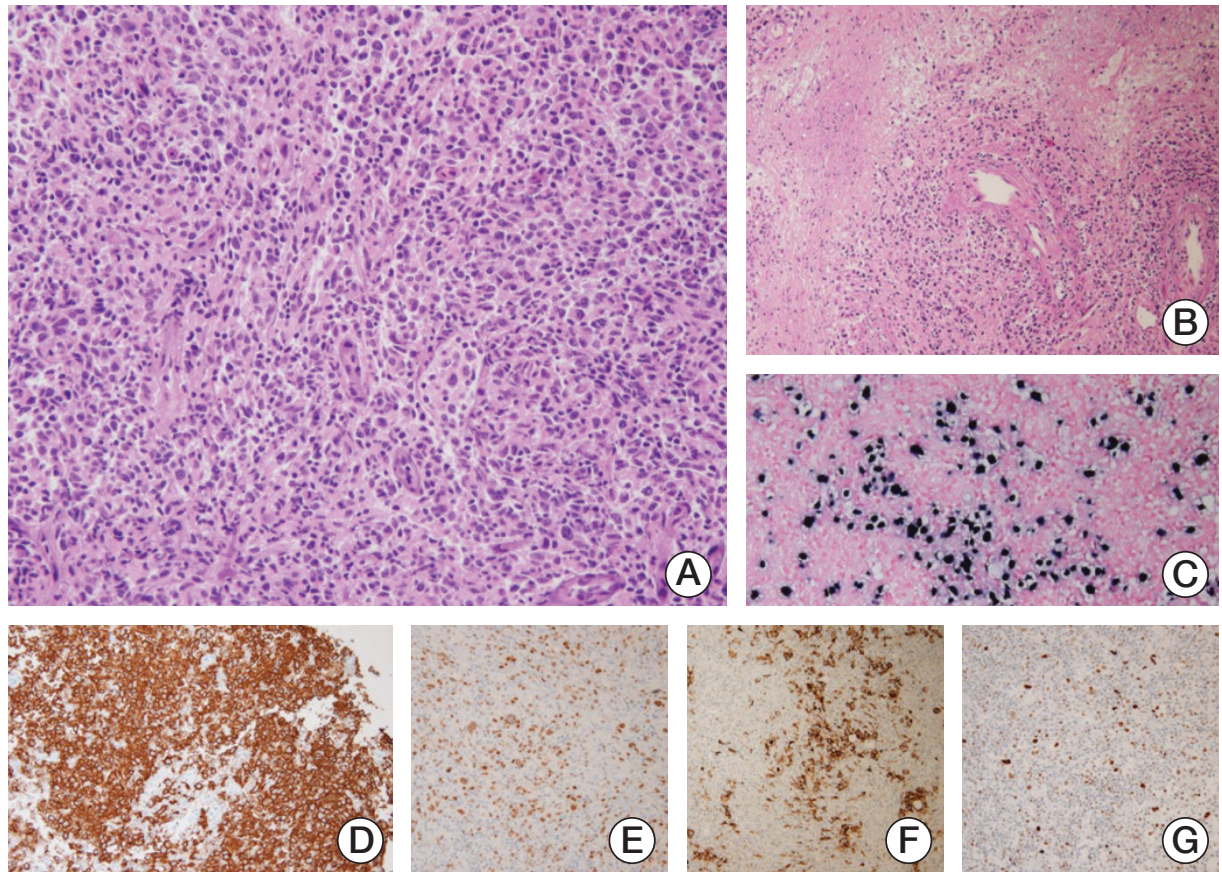

Fig. 2. (A) Diffuse large atypical lymphoid cell infiltrates (×200). (B) Focal destructive vascular invasion with surrounding necrosis $(\times 100)$. (C) Neoplastic lymphocytes were positive for Epstein-Barr virus encoded RNA on in situ hybridization $(\times 100)$. (D-G) The lymphocytes were diffusely immunoreactive for CD20 (D, $\times 200)$ and multiple myeloma oncogen-1 $(\mathrm{E}, \times 200)$, and showed patchy positivity for CD30 $(\mathrm{F}, \times 100)$ and $\mathrm{p} 53(\mathrm{G}, \times 200)$.
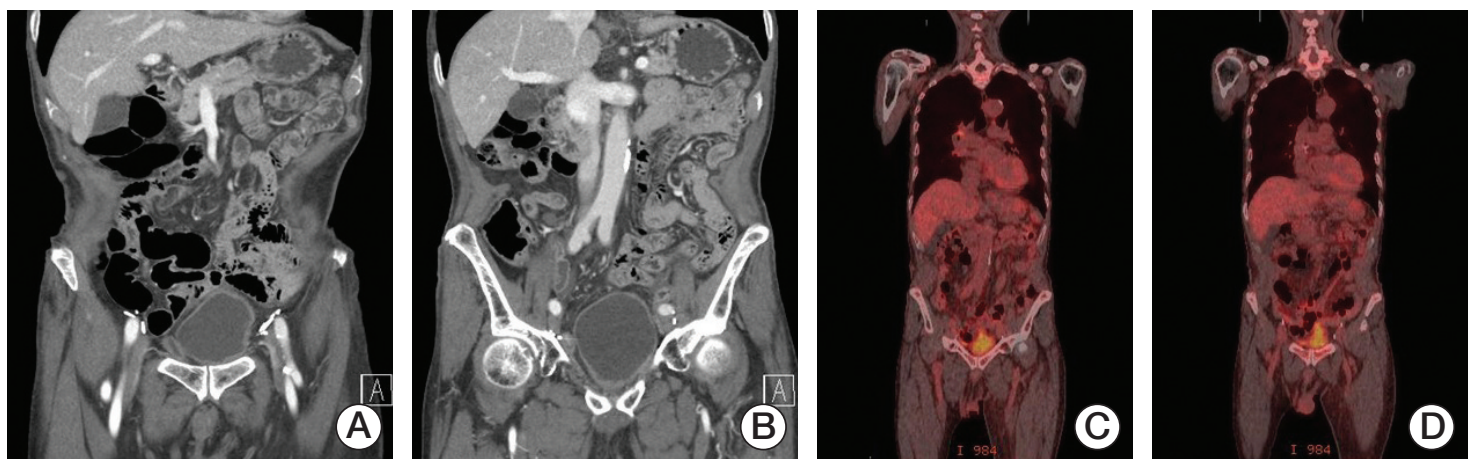

Fig. 3. Follow-up computed tomography scan after transurethral surgery and six cycles of "R-CHOP" chemotherapy showed disappearance of neobladder lesion (A) and multiple retroperitoneal lymphadenopathies (B). Follow-up positron emission tomography computed tomography showed no ${ }^{18} \mathrm{~F}$-fluorodeoxyglucose accumulation in ileal neobladder (C) and retroperitoneal lymph nodes (D), which was previously noted.

$\mathrm{mg} / \mathrm{m}^{2} \mathrm{D} 1$, and prednisone $100 \mathrm{mg}$ D1-5 (R-CHOP) for 21 days/cycle. After six cycles of chemotherapy, follow-up CT and PET-CT scan showed disappearance of neobladder lesion and multiple retroperitoneal lymphadenopathies
(Fig. 3). The patient's condition is good up to 2 months after finishing the chemotherapy, and he is under regular followup.

The study protocol was approved by the Institutional 
Review Board of the Eulji University Hospital (No. 2018-11003). The study patient was informed as to the purpose of the study and provided his consent.

\section{Discussion}

To our knowledge, this is the first report of EBV-positive DLBCL-CI arising in an ileal neobladder. To date, only two cases $[2,3]$ were reported with regards to malignant lymphoma arising in ileal conduit or ileal neobladder after radical cystectomy. However, the presence of EBV and lymphadenopathy was not detected in the prior DLBCL cases [2,3].

The major clinicopathological features of DLBCL-CI were adopted by the 2008 World Health Organization classification criteria as follows: occurrence in an enclosed environment, association with prolonged chronic inflammation, morphological features of DLBCL, positivity for B-cell immunophenotype, and positivity for EBV with type III latency [1]. The DLBCL-CI criteria rely primarily on clinical settings that prolonged inflammation within enclosed spaces induces the accumulation of cytokines such as interleukin (IL)-6 and IL-10, thereby promoting the proliferation of EBVinfected B cells. DLBCL-CI has been reported in association with previous artificial manipulation such as metallic implant, prosthetic heart valves, synthetic vascular Dacron grafts, surgical mesh implant which could induce prolonged immune reaction against foreign material $[7,8]$. While our patient had neither a history of inflammatory bowel disease nor immunosuppressive therapy, ileal neobladder with metallic clips, as an artificially manipulated enclosed organ, could be vulnerable to malignant transformation associated with prolonged inflammation due to chronic exposure to metallic clips and harmful urine components $[2,3]$.

EBV, a ubiquitous gamma herpes virus, is usually acquired silently early in life and carried thereafter as an asympto- matic infection of the B lymphoid system [9]. In our case, blood tests and PCR result for EBV indicate remote infection. Disruption of the finely regulated balance between virus and host immune system can result in EBV-associated lymphoproliferative disorders (LPDs). While there is still uncertainty on the spectrum of EBV-associated B-cell LPDs, EBV has been involved in the occurrence of Burkitt lymphoma, lymphomas with immunodeficiency, DLBCL not otherwise specified, DLBCL-CI, and lymphomatoid granulomatosis [10]. In particular, the diagnosis of lymphomatoid granulomatosis was considered as a differential diagnosis in relation to the histological features of lympho-histiocytic infiltration with epithelioid reaction and angiodestruction. However, because our patient did not have pulmonary involvement, the diagnosis of DLBCL-CI was more appropriate rather than lymphomatoid granulomatosis [11].

Although there is no convincing report regarding prognostic value of EBV positivity in specific DLBCL-CI, EBV-positive DLBCL has been reported to be associated with poor clinical outcomes [12,13]. For example, Montes-Moreno et al. [13] reported that EBV-positive DLBCL of the elderly had poor survival with estimated 2-year overall survival rate of $40 \%$ and 2-year progression-free survival of $36 \%$. Thus, although our patient with EBV-positive DLBCL-CI showed complete remission immediately after six cycles of chemotherapy, further long-term follow-up will be mandatory.

In conclusion, this case is the first report of the development of EBV-positive DLBCL-CI in an ileal neobladder. Although DLBCL is very rare in ileal conduit or ileal neobladder after radical cystectomy, clinicians should consider the possibility of lymphoma associated with chronic inflammation in patients presented with hematuria during longterm follow-up after bladder reconstruction.

\section{Conflicts of Interest}

Conflict of interest relevant to this article was not reported.

\section{References}

1. Dojcinov SD, Fend F, Quintanilla-Martinez L. EBV-positive lymphoproliferations of B- T- and NK-cell derivation in nonImmunocompromised hosts. Pathogens. 2018;7:E28.

2. Ota S, Iwai T, Kawaji Y, Fujino T, Ohshiro M, Hirakawa Y, et al. A treatment refractory CD30-positive diffuse large B cell lymphoma in the ileal neobladder. Ann Hematol. 2017;96: 683-4.

3. Muta T, Nakaike T, Fujisaki T, Shiraishi T, Ohshima K. Diffuse large B-cell lymphoma arising primarily at the stoma after bladder reconstruction using ileal conduit. Intern Med. 2012; 51:643-6.

4. Nakatsuka S, Yao M, Hoshida Y, Yamamoto S, Iuchi K, Aozasa K. Pyothorax-associated lymphoma: a review of 106 cases. J Clin Oncol. 2002;20:4255-60.

5. International Non-Hodgkin's Lymphoma Prognostic Factors Project. A predictive model for aggressive non-Hodgkin's lym- 
pho-ma. N Engl J Med. 1993;329:987-94.

6. Jaffe ES. The 2008 WHO classification of lymphomas: implications for clinical practice and translational research. Hematology Am Soc Hematol Educ Program. 2009:523-31.

7. Loong F, Chan AC, Ho BC, Chau YP, Lee HY, Cheuk W, et al. Diffuse large B-cell lymphoma associated with chronic inflammation as an incidental finding and new clinical scenarios. Mod Pathol. 2010;23:493-501.

8. Sanchez-Gonzalez B, Garcia M, Montserrat F, Sanchez M, Angona A, Solano A, et al. Diffuse large B-cell lymphoma associated with chronic inflammation in metallic implant. J Clin Oncol. 2013;31:e148-51.

9. Taylor GS, Long HM, Brooks JM, Rickinson AB, Hislop AD. The immunology of Epstein-Barr virus-induced disease. Annu Rev Immunol. 2015;33:787-821.
10. Kim HJ, Ko YH, Kim JE, Lee SS, Lee H, Park G, et al. EpsteinBarr virus-associated lymphoproliferative disorders: review and update on 2016 WHO classification. J Pathol Transl Med. 2017;51:352-8

11. Colby TV. Current histological diagnosis of lymphomatoid granulomatosis. Mod Pathol. 2012;25 Suppl 1:S39-42.

12. Lu TX, Liang JH, Miao Y, Fan L, Wang L, Qu XY, et al. EpsteinBarr virus positive diffuse large B-cell lymphoma predict poor outcome, regardless of the age. Sci Rep. 2015;5:12168.

13. Montes-Moreno S, Odqvist L, Diaz-Perez JA, Lopez AB, de Villambrosia SG, Mazorra F, et al. EBV-positive diffuse large $\mathrm{B}$-cell lymphoma of the elderly is an aggressive post-germinal center B-cell neoplasm characterized by prominent nuclear factor-kB activation. Mod Pathol. 2012;25:968-82. 